\title{
Uniqueness of functions with its shifts or difference operators
}

\author{
RAJ SHREE DHAR \\ Higher Education Department, J and K Government \\ Jammu and Kashmir,INDIA \\ dhar.rajshree@jk.gov.in
}

October 15, 2018

\begin{abstract}
It is shown that if a non-constant meromorphic function $f(z)$ is of finite order and shares certain values with its shifts/difference operators then $f(z)$ coincides with that particular shift/difference operator.
\end{abstract}

Mathematics Subject Classification:

2010 : Primary 30D35. Secondary 30D30, 39A10.

Keywords : Meromorphic function, Shared values, Nevanlinna theory, Shifts, Difference Operator.

\section{INTRODUCTION AND DEFINITIONS:}

A meromorphic (respectively entire) function always means a non-constant function meromorphic (respectively analytic) in the complex plane. Nevanlinna theory of value distribution is concerned with the density of points where a meromorphic function takes a certain value in the complex plane. It is also assumed that the reader is familiar with the basic concepts of Nevanlinna Theory, see e.g. ([1],[2]), such as the characteristic function $\mathrm{T}(\mathrm{r}, \mathrm{f})$, proximity function $\mathrm{m}(\mathrm{r}, \mathrm{f})$, counting function $\mathrm{N}(\mathrm{r}, \mathrm{f})$ and so on. In addition, $\mathrm{S}(\mathrm{r}, \mathrm{f})$ denotes any quantity that satisfies the condition that $S(r, f)=o(T(r, f))$ as $r$ tends to infinity outside of a possible exceptional set of finite logarithimic measure. In the sequel, a meromorphic function a(z)is called a small function with respect to $\mathrm{f}$ if and only if $\mathrm{T}[\mathrm{r}, \mathrm{a}(\mathrm{z})]=\mathrm{o}(\mathrm{T}(\mathrm{r}, \mathrm{f}))$ as $\mathrm{r}$ tends to infinity outside of a possible exceptional set of finite logarithimic measure. We denote by $\mathrm{S}(\mathrm{f})$, the family of all such small meromorphic functions.

We say that two meromorphic functions $\mathrm{f}$ and $\mathrm{g}$ share the value a (belonging to extended complex plane) CM (IM)

provided that

$$
f(z) \equiv a
$$


if and only if

$$
g(z) \equiv a
$$

counting multiplicity (ignoring multiplicity).

We recall some of the basic definitions of deficiencies of Nevanlinna theory:

$$
\begin{aligned}
& \delta(a, f)=1-\overline{\lim r} \rightarrow \infty \frac{N(r, a)}{T(r, f)} \\
& \Theta(a, f)=1-\overline{\lim r} \rightarrow \infty \frac{\bar{N}(r, a)}{T(r, f)}
\end{aligned}
$$

DEFINITION 1 :

Let $\mathrm{c}$ be a non-zero complex costant then for a meromorphic function $\mathrm{f}(\mathrm{z})$, we define its shift by $\mathrm{f}(\mathrm{z}+\mathrm{c})$ and its difference operator by

$$
\begin{gathered}
\Delta_{c} f(z)=f(z+c)-f(z), \\
\Delta_{m c} f(z)=f(z+m c)-f(z),
\end{gathered}
$$

where $\mathrm{m}$ is a positive integer

$$
\Delta_{c}^{n} f(z)=\Delta_{c}^{n-1}\left(\Delta_{c} f(z)\right)
$$

$\mathrm{n} \in \mathbf{N}, n \geq 2$,

$$
=\sum_{k=0}^{n} \frac{(-1)^{k} \cdot n !}{k ! .(n-k) !} f(z+\overline{n-k} \cdot c) \text {. }
$$

In particular,

$$
\Delta_{c}^{n} f(z)=\Delta^{n} f(z)
$$

for $\mathrm{c}=1$.

We define Differential - difference Monomial as

$$
M[f]=\prod_{i=0}^{k} \prod_{j=0}^{m}\left[f^{(j)}\left(z+c_{i j}\right)\right]^{n} i j
$$

where $c_{i j}$ are complex constants, and $n_{i j}$ are natural numbers , $\mathrm{i}=0,1, \ldots$ , $\mathrm{k}$ and $\mathrm{j}=0,1, \ldots, \mathrm{m}$.

Then the degree of $\mathrm{M}[\mathrm{f}]$ will be the sum of all the powers in the product on the right hand side.

DEFINITION 2[3] : Let

$$
M_{1}[f], M_{2}[f], \ldots
$$

denote the distinct monomials in $\mathrm{f}$, and

$$
a_{1}(z), a_{2}(z), \ldots
$$


be the small meromorphic functions including complex numbers then

$$
P[f]=P[z, f]=\sum_{j \in \Delta} a_{j}(z) \cdot M_{j}[f]
$$

where $\Delta$ is a finite set of multi- indices, $a_{j}(z)$ are small functions of $\mathrm{f}, M_{j}[f]$ are differential- difference monomials,

will be called a differential- difference polynomial in $\mathrm{f}$, which is a finite sum of products of $\mathrm{f}$, derivatives of $\mathrm{f}$, their shifts, and derivatives of its shifts. $\mathrm{We}$ define the total degree $\mathrm{d}$ of $\mathrm{P}[\mathrm{z}, \mathrm{f}]$ in $\mathrm{f}$ as

$$
d=\underbrace{\operatorname{Max}}_{j \in \Delta} . d_{M_{j}} .
$$

If all the terms in the summation of $\mathrm{P}[\mathrm{f}]$ have same degrees, then $\mathrm{P}[\mathrm{f}]$ is known as homogeneous differential- difference polynomial. Usually, we take $\mathrm{P}[\mathrm{f}]$ such that $\mathrm{T}(\mathrm{r}, \mathrm{P}) \neq S(r, f)$.

Linear Difference Polynomial is defined as the Difference polynomial of degree one e.g.

$$
\Delta_{c}^{n} f(z)
$$

The classical result due to Nevanlinna theory of meromorphic functions is the five point theorem i.e. if two non-constant meromorphic functions $\mathrm{f}$ and $\mathrm{g}$ share five distinct values ignoring multiplicities(IM) then

$$
f(z) \equiv g(z)
$$

The number 5 is best possible. If the number of shared values is decreased, then the additional assumptions on value distribution needs to be introduced in order to obtain uniqueness.

Uniqueness Theory of Meromorphic functions is an important part of Nevanlinna Theory. Recently number of papers have focussed on the Nevanlinna Theory with respect to difference operators. Then many authors started to investigate the uniqueness of meromorphic functions sharing values with their shifts or difference operators.

\section{SECTION 1}

Korhonen and Halburd ([4],[5]) gave direction to study the uniqueness of $f(z)$ and its shift $\mathrm{f}(\mathrm{z}+\mathrm{c})$, where $\mathrm{c}$ is a non-zero complex constant. In 2009, Heitokangas et al [6] started to consider the value sharing problems for the shifts of meromorphic functions and obtained many results as following:

THEOREM A[6]:

a. Let $\mathrm{f}$ be a meromorphic function of finite order and let $\mathrm{c}$ be a non- zero 
complex constant, then

If $\mathrm{f}(\mathrm{z})$ and $\mathrm{f}(\mathrm{z}+\mathrm{c})$ share 3 distinct periodic small functions with period c CM, then $f(z)$ is periodic with period $c$.

b. In case of $f(z)$ to be entire function, if $f(z)$ and $f(z+c)$ share 2 distinct periodic small functions with period c CM, then $\mathrm{f}(\mathrm{z})$ is periodic with period $\mathrm{c}$.

The following consequence of the above theorem is IM (Ignoring multiplicity) analogue of it.

\section{THEOREM B[6]:}

a. Let $\mathrm{f}$ be a meromorphic function of finite order and let $\mathrm{c}$ be a non- zero complex constant, then

If $\mathrm{f}(\mathrm{z})$ and $\mathrm{f}(\mathrm{z}+\mathrm{c})$ share 3 distinct periodic small functions with period $\mathrm{c} \mathrm{IM}$, and if

$$
\overline{\lim } r \rightarrow \infty \frac{N(r, 0, f)}{T(r, f)}<\frac{1}{2}
$$

then $f(z)$ is periodic with period $c$.

b. In case of entire function, part a. holds when number of shared values is 2 along with other conditions.

Recently in 2016, X.M.Li et al. [7] considered sharing four values with their shifts as follows:

THEOREM C[7]:

a. Let $f$ be a meromorphic function of finite order and let $c$ be a non- zero complex constant, then

if $\mathrm{f}(\mathrm{z})$ and $\mathrm{f}(\mathrm{z}+\mathrm{c})$ share $0,1, \mathrm{c} \mathrm{IM}$, and share $\infty \mathrm{CM}$ where $\mathrm{c}$ is finite and not equal to 0,1 . Then $f(z+c)$ is equal to $f(z)$ for all complex $z$.

$b$. Let $f$ be a non-constant entire function of finite order and let $c$ be a nonzero complex constant, then

if $f(z)$ and $f(z+c)$ share $a(z), b(z)$ IM, where $a(z), b(z)$ are two distinct small functions of $f(z)$ such that $a(z), b(z) \neq \infty$. Then $f(z+c)$ is equal to $f(z)$.

Some existing uniqueness results on the cases when $\mathrm{g}$ is derivative/ differential polynomial/ shifts of $\mathrm{f}$ has been obtained, see e.g. ([8],[9],[10],[11]).

In this paper, we consider the case of uniqueness of $f(z)$ with its shift when they share 3 distinct non-zero, finite values(or small functions) IM, with additional conditions as follows. Before proceeding to the main results, let us give some examples.

EXAMPLES: 
1. Let $\mathrm{f}(\mathrm{z})=\sin ^{2} z(1-\cos z), c=\pi$ then $\mathrm{f}(\mathrm{z}+\mathrm{c})=\sin ^{2} z(1+\cos z)$.

Here $\mathrm{f}(\mathrm{z})$ and $\mathrm{f}(\mathrm{z}+\mathrm{c})$ share $0 \mathrm{IM}$ and $1 \mathrm{CM}$ and $\mathrm{f}(\mathrm{z}) \neq f(z+c)$, where $\mathrm{f}$ is entire.

2. Let $\mathrm{f}(\mathrm{z})=\left(1+e^{i z}\right)^{2}\left(1-e^{i z}\right), c=\pi$ then $\mathrm{f}(\mathrm{z}+\mathrm{c})=\left(1-e^{i z}\right)^{2}\left(1+e^{i z}\right)$.

Here $\mathrm{f}(\mathrm{z})$ and $\mathrm{f}(\mathrm{z}+\mathrm{c})$ share 0 IM and $1 \mathrm{CM}$ and $\mathrm{f}(\mathrm{z}) \neq f(z+c)$, where $\mathrm{f}$ is entire.

3. Let $\mathrm{f}(\mathrm{z})=(\sec \mathrm{z}-1)(\sec \mathrm{z}+1)^{2}, c=\pi$.

Here $\mathrm{f}(\mathrm{z})$ and $\mathrm{f}(\mathrm{z}+\mathrm{c})$ share $0,-1$ IM and $\infty \mathrm{CM}$ and $\mathrm{f}(\mathrm{z}) \neq f(z+c)$, where $\mathrm{f}$ is meromorphic.

4. Let $\mathrm{f}(\mathrm{z})=\tan ^{2} z, c=\pi / 2$ then $\mathrm{f}(\mathrm{z}+\mathrm{c})=\cot ^{2} z$.

Here $\mathrm{f}(\mathrm{z})$ and $\mathrm{f}(\mathrm{z}+\mathrm{c})$ share 1 and $-1 \mathrm{CM}$ and $\mathrm{f}(\mathrm{z}) \neq f(z+c)$, where $\mathrm{f}$ is meromorphic.

5. Let $\mathrm{f}(\mathrm{z})=\sin ^{2} z, c=\pi / 2$ then $\mathrm{f}(\mathrm{z}+\mathrm{c})=\cos ^{2} z$.

Here $\mathrm{f}(\mathrm{z})$ and $\mathrm{f}(\mathrm{z}+\mathrm{c})$ share $1 / 2 \mathrm{CM}$ and $\mathrm{f}(\mathrm{z}) \neq f(z+c)$, where $\mathrm{f}$ is entire.

6. Let $\mathrm{f}(\mathrm{z})=\mathrm{e}^{\sin z}, c=\pi$ then $\mathrm{f}(\mathrm{z})$ and $\mathrm{f}(\mathrm{z}+\mathrm{c})$ share $0,1,-1, \infty \mathrm{CM}$ and $\mathrm{f}(\mathrm{z}) \neq f(z+c)$.

The result is not satisfied because of infinite order of $f$. Therefore $f$ should be of finite order.

\section{Remark}

From above examples, we can guess that for a non-constant meromorphic function $\mathrm{f}(\mathrm{z})$, if $\mathrm{f}(\mathrm{z})$ and $\mathrm{f}(\mathrm{z}+\mathrm{c})$ share 3 distinct non-zero, finite values( or small functions) IM, they must coincide. By example 4 above, the number 3 is best possible. And for entire function $\mathrm{f}(\mathrm{z})$ the number of shared values must be 2 , and by example 5 the number 2 is best possible.

\section{MAIN RESULTS:}

\section{THEOREM 1.1:}

i. Let $\mathrm{f}(\mathrm{z})$ be a non-constant meromorphic function of finite order. If $\mathrm{f}(\mathrm{z})$ and $\mathrm{f}(\mathrm{z}+\mathrm{c})$ share 3 distinct small functions $\mathrm{a}(\mathrm{z}), \mathrm{b}(\mathrm{z}), \mathrm{c}(\mathrm{z})$ IM such that $\mathrm{a}(\mathrm{z})$, $\mathrm{b}(\mathrm{z}), \mathrm{c}(\mathrm{z}) \neq 0, \infty$ identically and $\mathrm{E}(0, \mathrm{f}(\mathrm{z})) \subseteq E(0, f(z+c)), E(\infty, \mathrm{f}(\mathrm{z}+\mathrm{c})) \subseteq$ $E(\infty, \mathrm{f}(\mathrm{z}))$, then $\mathrm{f}(\mathrm{z}) \equiv \mathrm{f}(\mathrm{z}+\mathrm{c})$ for all complex $\mathrm{z}$, where $\mathrm{E}(0, \mathrm{f}(\mathrm{z}))$ is the set of zeros of $f(z)$.

ii. Let $f(z)$ be a transcendental entire function of finite order. If $f(z)$ and $\mathrm{f}(\mathrm{z}+\mathrm{c})$ share 2 distinct small functions $\mathrm{a}(\mathrm{z}), \mathrm{b}(\mathrm{z})$ such that $\mathrm{a}(\mathrm{z}), \mathrm{b}(\mathrm{z}) \neq 0, \infty$ identically and $\mathrm{E}(0, \mathrm{f}(\mathrm{z})) \subseteq E(0, f(z+c))$, then $\mathrm{f}(\mathrm{z}) \equiv \mathrm{f}(\mathrm{z}+\mathrm{c})$ for all complex $\mathrm{z}$.

THEOREM 1.2:

i. Let $f(z)$ be a non-constant meromorphic function of finite order. If $f(z)$ and 
$\mathrm{f}(\mathrm{z}+\mathrm{c})$ share one small function $\mathrm{a}(\mathrm{z}) \mathrm{CM}$ such that $\mathrm{a}(\mathrm{z}) \neq 0, \infty$, then $\mathrm{f}(\mathrm{z}) \equiv$ $\mathrm{f}(\mathrm{z}+\mathrm{c})$ for all complex z provided that $\delta(0, f)+\delta(\infty, \mathrm{f})>1$.

ii. Let $f(z)$ be a non-constant entire function of finite order. If $f(z)$ and $\mathrm{f}(\mathrm{z}+\mathrm{c})$ share one small function $\mathrm{a}(\mathrm{z}) \mathrm{CM}$ such that $\mathrm{a}(\mathrm{z}) \neq 0, \infty$, then $\mathrm{f}(\mathrm{z}) \equiv$ $\mathrm{f}(\mathrm{z}+\mathrm{c})$ for all complex z provided that $\delta(0, f)>0$.

\section{SECTION 2}

In this section, we consider the uniqueness of non-periodic function with its difference operator $\Delta_{c} f(z)=f(z+c)-f(z)$,

In 2016, Li and $\mathrm{Yi}[7]$ considered the case where entire function $\mathrm{f}(\mathrm{z})$ and $\Delta_{c} f(z)$ share three values IM and obtained the following:

\section{THEOREM D[7]:}

a. Let $\mathrm{f}$ be a non-constant meromorphic function of finite order and $\mathrm{c}$ be a non-zero complex number. Suppose that $\mathrm{f}$ and $\Delta_{c} f(z)$ share 3 distinct finite values IM and share $\infty, \mathrm{CM}$ then $\Delta_{c} f(z)=f(z)$, for all complex z.

b. Let $\mathrm{f}$ be a non-constant meromorphic function of finite order and $\mathrm{c}$ be a non-zero complex number. Suppose that $\mathrm{f}$ and $\Delta_{c} f(z)$ share 4 distinct finite values IM then $\Delta_{c} f(z)=f(z)$, for all complex z.

c. Let $\mathrm{f}$ be a non-constant entire function of finite order and $\mathrm{c}$ be a non-zero complex number. Suppose that $\mathrm{f}$ and $\Delta_{c} f(z)$ share 3 distinct finite values IM then $\Delta_{c} f(z)=f(z)$, for all complex z.

In 2017, Sheng Li et. al [10] considered the case where entire function $\mathrm{f}(\mathrm{z})$ and $\Delta_{c}^{n} f(z)$ share one value CM and one value IM with an extra condition and obtained the following:

THEOREM E[10]:

Let $\mathrm{f}$ be a non-constant entire function of finite order and $\mathrm{c}$ be a non-zero complex number. Suppose that $\mathrm{f}$ and $\Delta_{c}^{n} f(z)$ share 2 distinct complex constants a $\mathrm{CM}$ and $\mathrm{b}$ IM and if $\mathrm{N}(\mathrm{r}, \mathrm{a}, \mathrm{f}(\mathrm{z}))=\mathrm{T}(\mathrm{r}, \mathrm{f})+\mathrm{S}(\mathrm{r}, \mathrm{f})$ then

$$
\Delta_{c}^{n} f(z)=f(z),
$$

for all complex z.

In this paper, we prove that if meromorphic function $\mathrm{f}(\mathrm{z})$ and $\Delta_{c} f(z)$ share 0 , infinity $\mathrm{CM}$ and one small function $\mathrm{a}(\mathrm{z}) \mathrm{CM}$ such that $\mathrm{a}(\mathrm{z}) \neq 0, \infty$, then they will coincide as shown by the following results and we see that sharing of one non-zero and finite complex constant/ small function cannot be dropped as shown by following examples. 


\section{SOME EXAMPLES:}

1. Let $\mathrm{f}(\mathrm{z})=(\sec \mathrm{z}-1)(\sec \mathrm{z}+1)^{2}, c=\pi$ then $\Delta_{c} f(z)$ and $\mathrm{f}(\mathrm{z})$ share 0 $\mathrm{IM}$ and $\infty \mathrm{CM}$ and $\Delta_{c} f(z) \neq \mathrm{f}(\mathrm{z})$.

2.Let $f(z)=\frac{1}{\left(1+e^{z i}\right)^{2}\left(1-e^{z i}\right)}, \mathrm{c}=\pi$ then $\delta(0, f)+\delta(\infty, \mathrm{f})=1, \Delta_{c} f(z)$ and $\mathrm{f}(\mathrm{z})$ share share $0 \mathrm{CM}$ and $\infty \mathrm{IM}$ and $\Delta_{c} f(z) \neq \mathrm{f}(\mathrm{z})$.

3.Let $f(z)=(\sin \pi \mathrm{z})\left(\mathrm{e}^{z \log 2}\right) /(\cos 2 \pi z), \mathrm{c}=1$ then $\Delta_{c} f(z)$ and $\mathrm{f}(\mathrm{z})$ share 0 and $\infty \mathrm{CM}$ and $\Delta_{c} f(z) \neq \mathrm{f}(\mathrm{z})$.

4.Let $f(z)=\mathrm{e}^{z \log 2}$, here $\delta(0, f)+\delta(\infty, \mathrm{f})=2$ and $\Delta_{c} f(z)=f(z)$

\section{MAIN RESULTS:}

\section{THEOREM 2.1:}

i. Let $\mathrm{f}(\mathrm{z})$ be a non-constant meromorphic function of finite order and $\mathrm{c}$ be a non zero complex number. If for $\Delta_{c} f(z) \neq 0, \mathrm{f}(\mathrm{z})$ and $\Delta_{c} f(z)$ share $0, \infty \mathrm{CM}$ and one small function $\mathrm{a}(\mathrm{z})$ CM such that $\mathrm{a}(\mathrm{z}) \neq 0, \infty$ and then $\mathrm{f}(\mathrm{z}) \equiv \Delta_{c} f(z)$ for all complex $\mathrm{z}$.

ii. Let $\mathrm{f}(\mathrm{z})$ be a non-constant entire function of finite order. If for $\Delta_{c} f(z) \neq$ $0, \mathrm{f}(\mathrm{z})$ and $\Delta_{c} f(z)$ share 0 , one small functions a $(\mathrm{z})$ such that $\mathrm{a}(\mathrm{z}) \neq 0, \infty \mathrm{CM}$ , then $\mathrm{f}(\mathrm{z}) \equiv \Delta_{c} f(z)$ for all complex z.

\section{THEOREM 2.2:}

i. Let $\mathrm{f}(\mathrm{z})$ be a non-constant meromorphic function of finite order and $\mathrm{c}$ be a non-zero complex number.If for $\Delta_{c} f(z) \neq 0, \mathrm{f}(\mathrm{z})$ and $\Delta_{c} f(z)$ share 1 small function $\mathrm{a}(\mathrm{z})$ IM such that $\mathrm{a}(\mathrm{z}) \neq 0, \infty$ then $\mathrm{f}(\mathrm{z}) \equiv \Delta_{c} f(z)$ for all complex $\mathrm{z}$ provided $\delta(0, f)+\delta(\infty, \mathrm{f})>3 / 2$.

ii. Let $\mathrm{f}(\mathrm{z})$ be a non-constant entire function of finite order and $\mathrm{c}$ be a nonzero complex number.If for $\Delta_{c} f(z) \neq 0, \mathrm{f}(\mathrm{z})$ and $\Delta_{c} f(z)$ share 1 small function $\mathrm{a}(\mathrm{z})$ IM such that $\mathrm{a}(\mathrm{z}) \neq 0, \infty$ then $\mathrm{f}(\mathrm{z}) \equiv \Delta_{c} f(z)$ for all complex z provided $\delta(0, f)>1 / 2$.

Remark: From examples above, numbers 2 and 1 are best possible.

For the proof of the results we need the following lemmas:

LEMMA 1 ([5], Theorem 2.1): Let $\mathrm{f}$ be a non- constant meromorphic function of finite order and c be a non- zero complex constant, then

$$
m\left(r, \frac{f(z+c)}{f(z)}\right)=\mathrm{S}(\mathrm{r}, \mathrm{f}), m\left(r, \frac{\Delta_{c}^{n} f(z)}{f(z)-a}\right)=\mathrm{S}(\mathrm{r}, \mathrm{f})
$$


for all $\mathrm{r}$ outside a possible exceptional set of finite logarithmic measure.

LEMMA 2([4], Theorem 2.4): Let c be a non-zero complex constant, and let $\mathrm{f}$ be a meromorphic function of finite order such that $\Delta_{c} f$ is not identically zero. Let $\mathrm{q} \geq 2$, and let $a_{1}, a_{2}, \ldots, a_{q}$ be distinct meromorphic periodic small functions with period $\mathrm{c}$, then $\mathrm{m}(\mathrm{r}, \mathrm{f})+\sum_{k=1}^{q} m\left(r, \frac{1}{f-a_{k}}\right) \leq$ $2 \mathrm{~T}(\mathrm{r}, \mathrm{f})-2 \mathrm{~N}(\mathrm{r}, \mathrm{f})+\mathrm{N}\left(\mathrm{r}, \Delta_{c} f\right)-\mathrm{N}\left(\mathrm{r}, 1 / \Delta_{c} f\right)+\mathrm{S}(\mathrm{r}, \mathrm{f})$.

LEMMA 3(Clunie type lemma([12]): Let $\mathrm{f}(\mathrm{z})$ be a non- constant meromorphic function of finite order such that

$$
f^{n} P[z, f]=Q[z, f],
$$

where $P[z, f], Q[z, f]$ are differential-difference polynomials in $f$. If the degree of $\mathrm{Q}[\mathrm{z}, \mathrm{f}]$ as a polynomial in $\mathrm{f}$ and its shifts is at most $\mathrm{n}$, then

$$
m(r, P[z, f])=S(r, f) .
$$

\section{Corollary}

When we consider the uniqueness of function with its differential- difference monomials $\mathrm{M}[\mathrm{f}]$ or polynomials $\mathrm{P}[\mathrm{f}]$ as in definitions 1 and 2 , the result for linear difference polynomial holds as in section 2 replacing $\Delta_{c} f(z)$ by linear difference polynomial and by using Lemma 3 on the same grounds of section 2 . The result of uniqueness also holds for $f^{d}$ with its differential- difference monomials $\mathrm{M}[\mathrm{f}]$ or polynomials $\mathrm{P}[\mathrm{f}]$ where $\mathrm{d}$ is the corresponding degree, see[9].

\section{PROOF OF THEOREM 1.1:}

i. Suppose on the contrary, then

$$
f(z+c) \neq f(z) .
$$

Since $\mathrm{f}(\mathrm{z})$ and $\mathrm{f}(\mathrm{z}+\mathrm{c})$ share 3 distinct $\mathrm{a}(\mathrm{z}), \mathrm{b}(\mathrm{z}), \mathrm{c}(\mathrm{z})(\neq 0, \infty)$, therefore, by using Nevanlinna's Second Fundamental Theorem, Lemma 1 and the given conditions, we get:

$$
\begin{aligned}
\mathrm{T}(\mathrm{r}, \mathrm{f}) \leq \bar{N}\left(r, \frac{1}{f-a(z)}\right)+ & \bar{N}\left(r, \frac{1}{f-b(z)}\right)+\bar{N}\left(r, \frac{1}{f-c(z)}\right)+S(r, f) \\
\leq & \bar{N}\left(r, \frac{1}{f(z+c)-f(z)}\right)+S(r, f) \\
= & \bar{N}\left(r, 1, \frac{f(z+c)}{f(z)}\right)+S(r, f)
\end{aligned}
$$




$$
\begin{gathered}
\leq T\left(r, \frac{f(z+c)}{f(z)}\right)+S(r, f) \\
=N\left(r, \frac{f(z+c)}{f(z)}\right)+S(r, f) \\
=S(r, f)
\end{gathered}
$$

by using given conditions of the theorem, a contradiction.

Therefore,

$$
f(z+c) \equiv f(z) .
$$

ii. The case of entire functions follows in the same steps as in (i) taking $\bar{N}(r, f)=S(r, f)$.

\section{PROOF OF THEOREM 1.2:}

i. Suppose on the contrary, then

$$
f(z+c) \neq f(z) .
$$

Since $f(z)$ and $f(z+c)$ share one small function $a(z)$ CM such that $a(z) \neq 0, \infty$, therefore, by using Lemma 2 and the given conditions, we get:

$$
\begin{aligned}
& \quad \mathrm{T}(\mathrm{r}, \mathrm{f}) \leq N(r, f)+N\left(r, \frac{1}{f}\right)+N\left(r, \frac{1}{f-a(z)}\right)+N\left(r, \Delta_{c} f\right)-2 N(r, f)-N\left(r, \frac{1}{\Delta_{c} f}\right) \\
& +\mathrm{S}(\mathrm{r}, \mathrm{f}) \\
& \quad<T(r, f)+N\left(r, \frac{1}{\Delta_{c} f}\right)+\mathrm{N}\left(\mathrm{r}, \Delta_{c} f\right)-2 N(r, f)-N\left(r, \frac{1}{\Delta_{c} f}\right)+\mathrm{S}(\mathrm{r}, \mathrm{f}) \\
& \quad \text { (by using } \delta(0, f)+\delta(\infty, \mathrm{f})>1) . \\
& \quad \leq T(r, f)
\end{aligned}
$$

a contradiction. Therefore,

$$
f(z+c) \equiv f(z) .
$$

ii. Suppose on the contrary, then

$$
f(z+c) \neq f(z) .
$$

Since $f(z)$ and $f(z+c)$ share one small function $a(z) C M$ such that $a(z) \neq 0, \infty$, therefore, by using Lemma 2 and the given condition, we get: 


$$
\begin{aligned}
& \mathrm{T}(\mathrm{r}, \mathrm{f}) \leq N(r, f)+N\left(r, \frac{1}{f}\right)+N\left(r, \frac{1}{f-a(z)}\right)+N\left(r, \Delta_{c} f\right)-2 N(r, f)-N\left(r, \frac{1}{\Delta_{c} f}\right) \\
+ & \mathrm{S}(\mathrm{r}, \mathrm{f}) \\
& <T(r, f)+N\left(r, \frac{1}{\Delta_{c} f}\right)+\mathrm{N}\left(\mathrm{r}, \Delta_{c} f\right)-2 N(r, f)-N\left(r, \frac{1}{\Delta_{c} f}\right)+\mathrm{S}(\mathrm{r}, \mathrm{f})
\end{aligned}
$$

(by using $\delta(0, f)>0$ ).

$$
\leq T(r, f)+S(r, f),
$$

a contradiction. Therefore,

$$
f(z+c) \equiv f(z) .
$$

\section{PROOF OF THEOREM 2.1:}

i. Suppose on the contrary, then

$$
\Delta_{c} f \neq f .
$$

Since $\mathrm{f}$ and $\Delta_{c} f$ share $0, \infty \mathrm{CM}$ and also one small function a(z) $\mathrm{CM}$ such that $\mathrm{a}(\mathrm{z}) \neq 0, \infty$, therefore, by using Lemma 2 , we get:

$$
\begin{aligned}
& \mathrm{T}(\mathrm{r}, \mathrm{f}) \leq N(r, f)+N\left(r, \frac{1}{f-a(z)}\right)+N\left(r, \frac{1}{f}\right)+N\left(r, \Delta_{c} f\right)-2 N(r, f)-N\left(r, \frac{1}{\Delta_{c} f}\right) \\
+ & \mathrm{S}(\mathrm{r}, \mathrm{f}) \\
& =\mathrm{N}(\mathrm{r}, \mathrm{f})+\mathrm{N}\left(\mathrm{r}, \frac{1}{\Delta_{c} f-f}\right)+\mathrm{N}(\mathrm{r}, \mathrm{f})-2 \mathrm{~N}(\mathrm{r}, \mathrm{f})-\mathrm{N}\left(\mathrm{r}, \frac{1}{\Delta_{c} f}\right)+\mathrm{S}(\mathrm{r}, \mathrm{f}) \\
& =\mathrm{N}\left(\mathrm{r}, \frac{1}{\Delta_{c} f-f}\right)-\mathrm{N}\left(\mathrm{r}, \frac{1}{\Delta_{c} f}\right)+\mathrm{S}(\mathrm{r}, \mathrm{f}) \\
& =\mathrm{N}\left(\mathrm{r}, \frac{1}{\Delta_{c} f}\right)-\mathrm{N}\left(\mathrm{r}, \frac{1}{\Delta_{c} f}\right)+\mathrm{S}(\mathrm{r}, \mathrm{f}) \\
& =\mathrm{S}(\mathrm{r}, \mathrm{f})
\end{aligned}
$$

a contradiction. Therefore,

$$
\Delta_{c} f \equiv f .
$$

ii. The case of entire functions follows in the same steps as in (i) taking $\mathrm{N}(\mathrm{r}$, f) $=S(r, f)$.

\section{PROOF OF THEOREM 2.2:}

i. Suppose on the contrary, then

$$
\Delta_{c} f \neq f .
$$


Since $\mathrm{f}(\mathrm{z})$ and $\Delta_{c} f(z)$ share 1 small function $\mathrm{a}(\mathrm{z})$ IM such that $\mathrm{a}(\mathrm{z}) \neq 0, \infty$ therefore, by using Nevanlinna's Second Fundamental Theorem, Lemma 1 and the given conditions, we get:

$$
\begin{aligned}
& \mathrm{T}(\mathrm{r}, \mathrm{f}) \leq \bar{N}\left(r, \frac{1}{f}\right)+\bar{N}(r, f)+\bar{N}\left(r, \frac{1}{f-a(z)}\right)+S(r, f) \\
& <\frac{1}{2} T(r, f)+\bar{N}\left(r, \frac{1}{f-a(z)}\right)+S(r, f) \\
& =\frac{1}{2} T(r, f)+\bar{N}\left(r, \frac{1}{\Delta_{c} f-f}\right)+\mathrm{S}(\mathrm{r}, \mathrm{f}) \\
& \leq \frac{1}{2} T(r, f)+\bar{N}\left(r, \frac{\Delta_{c} f}{f}, 1\right)+\mathrm{S}(\mathrm{r}, \mathrm{f}) \\
& \leq \frac{1}{2} T(r, f)+N\left(r, \frac{\Delta_{c} f}{f}\right)+S(r, f) \\
& <\frac{1}{2} T(r, f)+\frac{1}{2} T(r, f)+S(r, f)
\end{aligned}
$$

a contradiction. Therefore,

$$
\Delta_{c} f \equiv f
$$

ii. The case of entire functions follows in the same steps as in (i) taking $\mathrm{N}(\mathrm{r}$, $\mathrm{f})=\mathrm{S}(\mathrm{r}, \mathrm{f})$.

\section{Conflict of interest:}

The author has no conflict of interest to declare.

\section{REFERENCES:}

[1]. Hayman, W.K.: Meromorphic functions- Clarenden Press, Oxford, 1964.

[2]. Yang C. C., Yi, H. X.: Uniqueness theory of meromorphic functions, Kluver Academic Press 2003.

[3]. Yang, C.C. and Laine,I.- On analogies between nonlinear difference and differential equations, Proceedings of the Japan Academy A, vol. 86, no. 1, pp. 1014,2010

[4]. Halburd, R.G., Korhonen, R.J.: Nevanlinna Theory for the Difference Operator.- Annales Academiae Scientiarum Fennicae Mathematica, Volumen 31, pp.463- 478, 2006.

[5]. Halburd, R.G., Korhonen, R.J.: Difference Analogue of the lemma on the logarithmic derivativewith applications to difference equations, J. Math. Anal Appl., 314, No. 2, pp.477-487, 2006.

[6]. Heittokangas, J., Korhonen, R., Laine, I., Reippo, J.: Uniqueness of meromorphic functions sharing values with their shifts.- Complex variables and Elliptic functions, vol. 56, Nos 1-4, 81- 92, 2011.

[7]. Li.,X.M., Yi, H.X.,: Meromorphic functions sharing 4 values with their operators or shifts, Bull. Korean Math.Soc., 53, no. 4, pp. 1213- 1235, 2016. 
[8]. Dhar, R. S.,: Uniqueness Theorems on Meromorphic functions and their difference operators,- Int. Jour. of Math. Analysis, Vol.7, No. 30, pp. 1489$1495,2013$.

[9]. Dhar, R. S.,: On Differential- Difference polynomials,- Int. J. of Cont. Math. Sciences, Vol. 11, no. 11, pp. 517- 531, 2016.

[10]. Li.,Sheng, : Uniqueness of entire functions sharing 2 values with their difference operators, Advances in difference equations 2017: 390.

[11]. Lu Feng, Wang Y., Xu J.: Entire functionssharing a small function with their two difference operators Advances in difference equations 2017: 216.

[12]. Laine, I., Yang, C.C.: Clunie Theorems for difference and q- differential polynomials.- J. London Math. Soc. 76(2), pp.556- 566, 2007. 